\title{
Modification of Cysteine Residue in Peptides by a Fluorescent Reagent for Complete Sequence Analyses Using Post-Source Decay Method in Matrix-Assisted Laser Desorption/Ionization Time-of-Flight Mass Spectrometry
}

\author{
Masatoshi Nakagawa, ${ }^{\text {a) }}$ Tohru Yamagaki, ${ }^{\text {) }}$ and Hiroshi Nakanishi*a)
}

(Received June 3, 2003; Accepted July 29, 2003)

\begin{abstract}
In the sequence analyses of antigen peptides from nucleoprotein in influenza virus, it was very difficult to obtain the sufficient numbers of product ions using the post-source decay (PSD) method in MALDI-TOF-MS. Fluorescent modification of the thiol group of the cysteine residue in the target peptides using 5-iodoacetamide fluorescein was introduced for the PSD measurement. The fluorescently-labeled peptides gave sufficient product ions for complete sequence analyses in the PSD spectrum, which leads to the complete sequence analyses of the peptides with cysteine residue.
\end{abstract}

\section{Introduction}

In the fields of proteome and proteomics, it is well known that identification of the sequence of peptides and proteins is one of important steps. Recently, matrix-assisted laser desorption/ionization time-of-flight mass spectrometry (MALDI-TOF-MS) has become to be used for this purpose. ${ }^{1-4)}$ Analysis of a peptide sequence using MALDI-TOF-MS requires mass selection of a specified peptide ion (the precursor ion) within the mass spectrometer, followed by fragmentation of the ion into product ions, in a process known as postsource decay (PSD). ${ }^{5)}$, 6) Since the product ions must derive exclusively from the precursor ion, analysis of the $m / z$ values of the product ions allows sequence determination of the parent peptide ion. Theoretically, peptides can be sequenced by analyzing the product ions produced by PSD fragmentation of the peptide during MALDI-TOF-MS. However, there are some peptides to be not amenable to full sequence interpretation using this method, because they are only partially fragmented during PSD. It is therefore desirable to improve the interpretability of the PSD fragment spectra produced using MALDI-TOF-MS. There are several studies about the improvement of the sensitivity of PSD fragmentation by the charge derivatization of peptide. $^{7)^{-11)}}$

Recently, we have preliminarily reported about the derivatization of the $\mathrm{N}$-terminal cysteine residue of peptide by fluorescent regent in MALDI-PSD method. ${ }^{12}$ The N-terminal cysteine residue in peptide from the nucleoprotein in influenza virus was derivatized at the thiol site by using 4 (5)-(iodoacetamide) fluorescein for which it is otherwise difficult to detect a full comple-

*a) Biological Information Research Center, National Institute of Advanced Industrial Science and Technology (Tsukuba Central 6, 1-1 Higashi, Tsukuba, Ibaraki 305-8566, Japan)

b) Department of Chemistry, School of Science, The University of Tokyo (7-3-1 Hongo, Bunkyo-ku, Tokyo 113-0033, Japan) ment of product ions. In the MALDI-TOF-MS experiment, the sensitivity of product ions in the fluorescently-labeled peptide in the PSD method was greatly improved.

In the present papers, we modified a thiol group of cysteine residue at the $\mathrm{C}$-terminal and middle positions of the target peptides by the fluorescent reagent in different reaction conditions and analyzed the PSD spectrum. The fluorescent modification at the C-terminal and middle positions of peptides gave satisfied results in the sequence analyses using MALDI-PSD method. This is a simple and straightforward technique for the sequence analyses of peptides with cysteine residue.

\section{Materials and Methods}

Peptides SDYEGRLC and SDYECRLI are mutated to the cysteine residue of a part of nucleoprotein (NP5057 ) in influenza virus. These antigen peptides bind to major histocompatibility complex class I molecules and play an important role in the immune response. ${ }^{13)}$ We chose these peptides as the target molecules for MALDI-PSD analyses. The sample peptides were synthesized chemically by solid-phase strategies on an automated multiple PSSM8 Shimadzu peptide synthesizer (Shimadzu, Kyoto, Japan) using Fmoc-chemistry. The protecting group and resin were cleaved from the synthesized compound by incubating with a cleavage cocktail (trifluoroacetic acid-anisole-1,2-ethanedithiol in a $94: 5: 1$ ratio) for $100 \mathrm{~min}$ at room temperature. The crude reactants were then washed in diethyl ether and freeze-dried. The peptides were isolated by reverse-phase HPLC (RP-HPLC) using the solvent $10 \mathrm{mM}$ $\mathrm{HCl}-$ acetonitrile in an $80: 20$ ratio, and dried. $0.1 \mathrm{mg}$ of these peptides was dissolved in $10 \mathrm{mM}$ sodium phosphate buffer, pH 7.0, at room temperature, respectively. Fluorescent derivative peptides were synthesized by mixing the peptide solution with $1 \mathrm{mg}$ 5-iodoacetamide fluorescein (Molecular Probes, Inc., Eugene, Or, USA) ${ }^{14)}$ dissolved in $N, N$-dimethylformamide (DMF) at room temperature for $2 \mathrm{~h}$. In our preliminary experiment of 
the derivatization of the $\mathrm{N}$-terminal cysteine of similar kind of antigen peptide, the reaction was done in acetonitrile solvent for 30 min at room temperature. ${ }^{12)}$ But in the case of the modification of cysteine residue at the C-terminal and middle positions of the peptides, DMF solvent in above mentioned condition gave us more appropriate products with a higher yield and less impurity.

The reaction solution was analyzed by MALDI-TOFMS after $2 \mathrm{~h}$ reaction. All peptides were now labeled with the fluorescent tag (data not shown), respectively. Fluorescently-labeled (Flu) peptides (Fig. 1) were isolated by RP-HPLC using the solvent $10 \mathrm{mM} \mathrm{HCl-aceto-}$ nitrile in a $70: 30$ ratio, and immediately analyzed by positive ion MALDI-TOF-MS. In the crude samples without the purification by HPLC, similar results of

(a)<smiles>[R15]c1ccc(NC(=O)CSc2cccc(-c3c4ccc(=O)cc-4oc4cc(O)ccc34)c2C(=O)O)cc1</smiles>

(b)

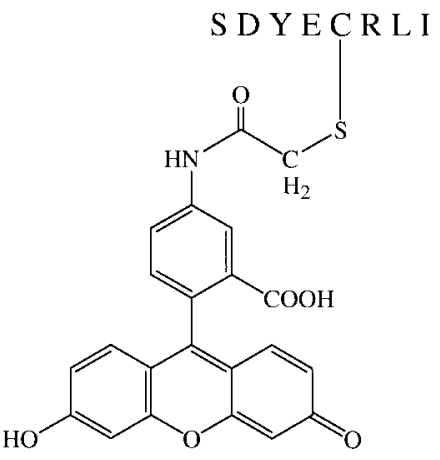

Fig. 1. Structure of fluorescently-labeled peptide (a) SDYEGRLC(Flu) and (b) SDYEC(Flu)RLI.
PSD measurement were obtained as in the purified samples. The sample solutions were mixed with the matrix solution of $30 \%$ acetonitrile saturated with $\alpha$ cyano-4-hydroxycinnamic acid (Aldrich, Milwaukee, WI, USA) on a sample plate, and then air dried on the plate. Final amount of peptide used in MS measurement was in picomole order. All MS experiments were performed in positive ion mode on an AXIMA-CFR instrument (Shimadzu, Kyoto, Japan), equipped with a reflectron. Sample ionization was performed by irradiation using a nitrogen laser emitting at $337 \mathrm{~nm}$. Reproducibility of the product ions of peptide in MALDIPSD spectra was in excellent degree.

\section{Results and Discussion}

The protonated molecule $[\mathrm{M}+\mathrm{H}]^{+}$of underivatized SDYEGRLC was observed at $m / z$ 944. This ion was used as the precursor ion in the PSD fragmentation measurements using MALDI-TOF-MS. The obtained spectrum is shown in Fig. 2. Only five sequential product ions $\mathrm{a}_{7}, \mathrm{~b}_{6}, \mathrm{z}_{7}, \mathrm{y}_{6}$, and $\mathrm{y}_{4}$ were clearly observed (notation of product ions was done as in the literature ${ }^{15)}$ ). These product ions were too insufficient to reveal fully the sequential analysis of the original peptide. Then, the fluorescently-labeled peptide SDYEGRLC(Flu) was synthesized for the sequential analysis of SDYEGRLC. To modify the C-terminal thiol group of cysteine residue in the peptide, 5-iodoacetoamide fluorescein was used in DMF at room temperature instead of acetonitrile used in our previous papers. ${ }^{12)}$ In the reaction conditions, the modification reaction finished more completely, and no side-reaction occurred. When the $[\mathrm{M}+\mathrm{H}]^{+}$ion of SDYEGRLC(Flu) $(\mathrm{m} / z$ 1331) was used as the precursor ion for PSD analysis, all sequential product ions in the y series, $\mathrm{y}_{7}$, $\mathrm{y}_{6}, \mathrm{y}_{5}, \mathrm{y}_{4}, \mathrm{y}_{3}, \mathrm{y}_{2}$, and $\mathrm{y}_{1}$ were observed (Fig. 3).

In addition to these y-series ions, seven product ions with the $m / z$ number of $\mathrm{y}_{n}-17$ or $\mathrm{y}_{n}-18(n=1-7)$ were also detected, which are shown in asterisk marks in the PSD spectrum in Fig. 3. It can be discussed that these product ions might occur due to the side reaction such

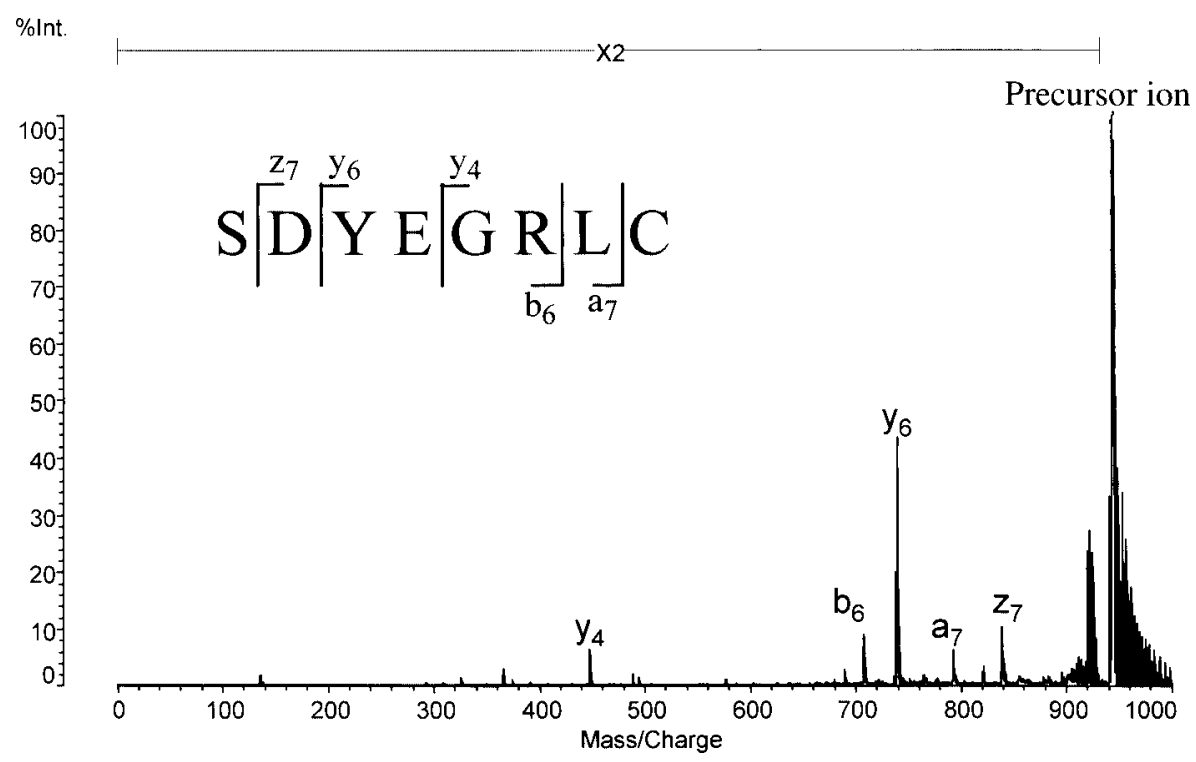

Fig. 2. PSD spectrum of SDYEGRLC peptide in MALDI-TOF-MS. The precursor ion of MS/MS experiment is at $m / z 944$. 


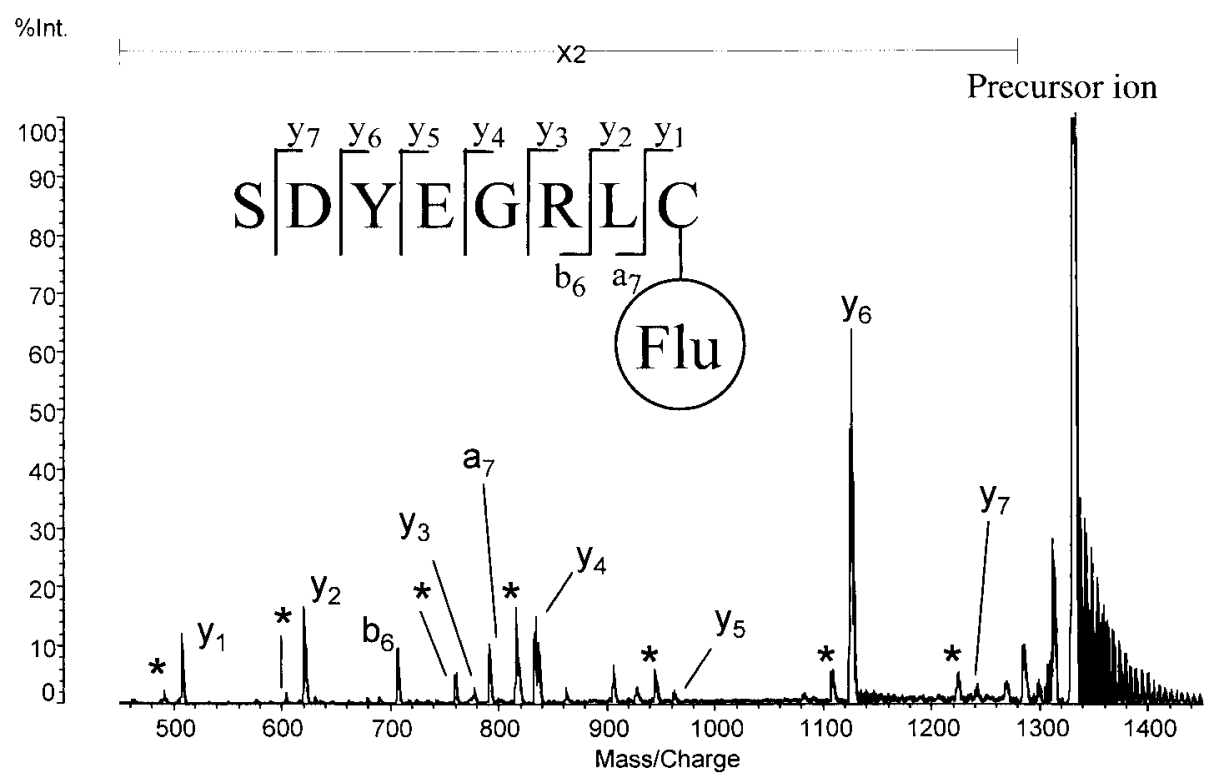

Fig. 3. PSD spectrum of SDYEGRLC(Flu) peptide in MALDI-TOF-MS. The precursor ion of MS/MS experiment is at $m / z$ 1331. Asterisk marks are product ions $\mathrm{y}_{n}-17$ and/or $\mathrm{y}_{n}-18$.

as the dehydration at the C-terminal amino acid residue and/or elimination of ammonia molecule during the PSD process, although the specific parts of these reactions could not be recognized.

Regarding to product ions of other series such as aand $\mathrm{b}$-series, two product ions, $\mathrm{a}_{7}$ and $\mathrm{b}_{6}$, were observed in both derivatized and underivatized peptides and there is no different fragmentation pattern in these series between the two peptides. These two peptides have a common arginine residue in the 6th position and the arginine could be the charge center in the PSD process. Thus, this may be a main reason for the occurrence of ions $a_{7}$ and $b_{6}$ in the peptides with relative large intensity around the arginine residue.

The main product ions produced by PSD measurements of underivatized and fluorescently-labeled peptide are shown schematically in Figs. 2 and 3, respectively. Comparison of Figs. 2 and 3 show that much more product ions are observed for the modified peptide than for the original peptide. Too incomplete product ions in a-, b-, y-, and $z$-series were observed for the underivatized peptide, and hence full sequencing of the peptide was not at all possible. In contrast, all product ions in the sequential y-series were successfully observed for the fluorescently-labeled peptide, and thus, the y-series is completely sufficient for satisfied interpretation of the peptide sequence. Furthermore, many product ions of $\mathrm{y}-17$ or $\mathrm{y}-18$ series were also observed in the fluorescently-labeled peptide and these additive data support the sequence analysis of the peptide.

For the analysis of the peptide with cysteine residue at the middle position, SDYECRLI peptide was chosen. In SDYECRLI, the protonated molecule $[\mathrm{M}+\mathrm{H}]^{+}$of underivatized peptide was observed at $m / z$ 999. This ion was used as the precursor ion in the PSD fragmentation measurements. The obtained spectrum is shown in Fig. 4. Five sequential product ions $\mathrm{a}_{7}, \mathrm{~b}_{7}, \mathrm{c}_{7}, \mathrm{~b}_{6}$, and $\mathrm{y}_{6}$ were clearly observed and shown schematically above left in Fig. 4. In addition, $\mathrm{b}_{8}-17$ and $\mathrm{b}_{6}-17$ ions were also detected. It was impossible to obtain the information of full sequence of the peptide by using these small kinds of product ions. Thus, the thiol group at cysteine residue of SDYECRLI was modified with 5-iodoacetoamide fluorescein as in the case of the C-terminal peptide. The $[\mathrm{M}+\mathrm{H}]^{+}$ion of SDYEC(Flu) RLI $(m / z 1386)$ was used as the precursor ion for PSD analysis (Fig. 5). The sequential product ions $\mathrm{a}_{8}, \mathrm{~b}_{8}, \mathrm{a}_{7}$, $\mathrm{b}_{7}, \mathrm{c}_{7}, \mathrm{a}_{6}, \mathrm{~b}_{6}, \mathrm{a}_{5}, \mathrm{~b}_{5}, \mathrm{y}_{7}, \mathrm{y}_{6}, \mathrm{y}_{5}$, and $\mathrm{y}_{4}$ were observed and shown schematically above left in Fig. 5. In addition, $\mathrm{b}_{8}-17, \mathrm{~b}_{7}-17, \mathrm{a}_{6}-17$, and $\mathrm{b}_{6}-17$ product ions were also detected and these supported the sequential analysis. Similarly in the case of the C-terminal peptide SDYEGRLC(Flu), other product ions such as $\mathrm{y}_{6}-17, \mathrm{y}_{5}-17$, and $\mathrm{y}_{4}-17$ were also detected, which was shown in Fig. 5 in asterisk marks. In this PSD analysis of fluorescentlylabeled peptide, thus the sequential analysis is performed completely.

It is possible that the increased sensitivity of the PSD measurement for the fluorescently-labeled peptide compared to the underivatized peptide results from improved energy transmission efficiency between the matrix and the peptide through the introduction of the fluorescent group. Alternatively, the difference may be explained by the addition of the positive charge center on the $[\mathrm{M}+\mathrm{H}]^{+}$ions of the peptides. The original peptides carry two positive charge centers, one on the $\mathrm{N}$-terminal amino group, and another on the side chain of the arginine residue. In the fluorescently-labeled peptides (Figs. 3 and 5), the same common product ions observed in spectrum of the original peptide were detected, and additionally many new product ions were obviously observed. Since the fluorescent moiety has the specific structural character with a large capacity against the protonation, these results suggest that the positively charged fluorescent group at the C-terminus or middle positions of the peptide is a more effective charge center than those of the arginine and $\mathrm{N}$ terminal residues in PSD fragmentation of the peptide. As the result, the numbers of charged product ions 


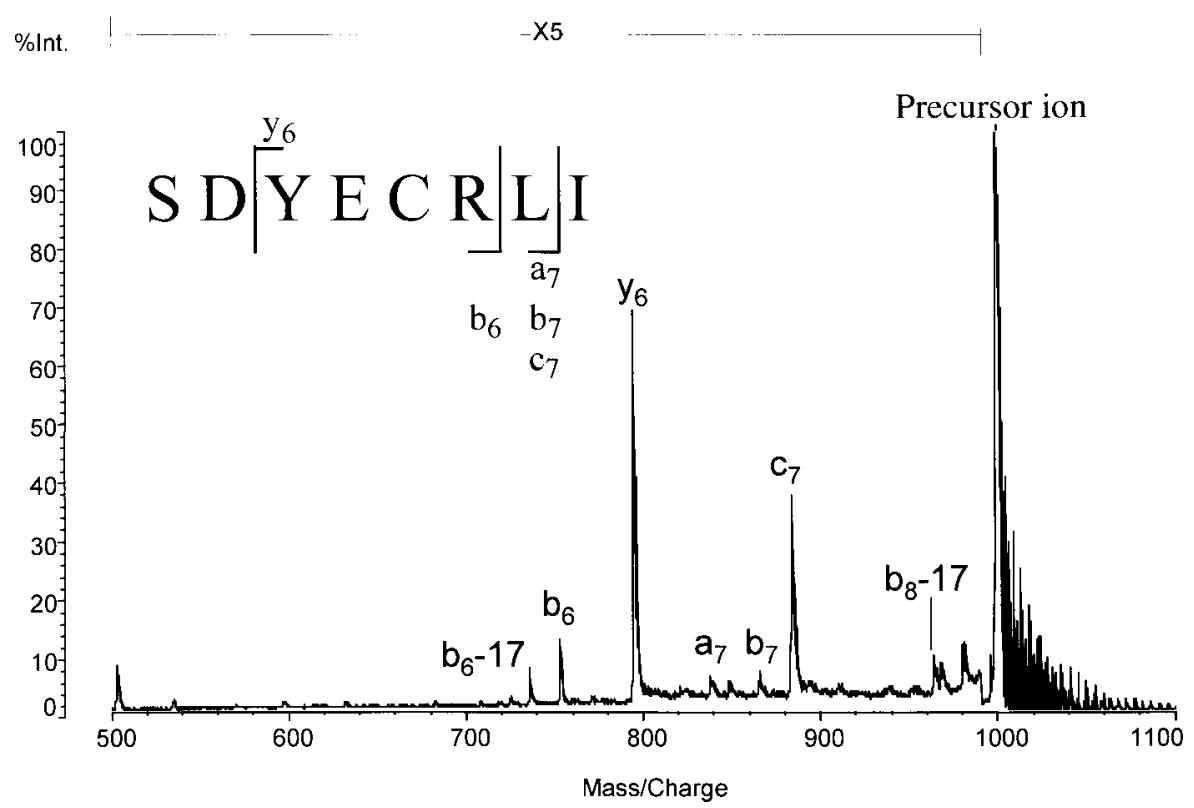

Fig. 4. PSD spectrum of SDYECRLI peptide in MALDI-TOF-MS. The precursor ion of MS/MS experiment is at $m / z 999$.

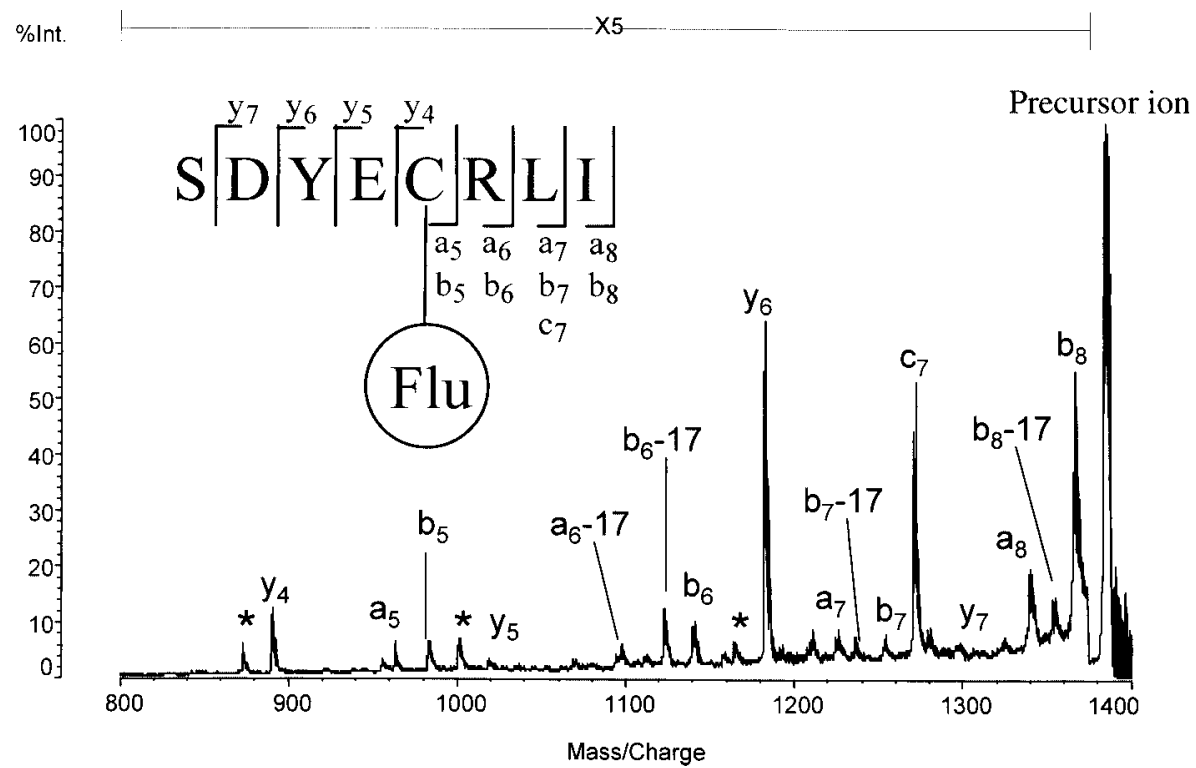

Fig. 5. PSD spectrum of SDYEC(Flu)RLI peptide in MALDI-TOF-MS. The precursor ion of MS/MS experiment is at $m / z$ 1386. Asterisk marks are product ions $\mathrm{y}_{n}-17$ and/or $\mathrm{y}_{n}-18$.

could increase by the fluorescent labeling.

Recently, we reported that fluorescent modification of a peptide with an N-terminal cysteine residue enhances the sensitivity of MALDI-TOF-MS for PSD analysis. $^{12)}$ In this paper, we report the results of the fluorescent modification of peptides with a cysteine residue in the C-terminal and the middle position. Although the fragmentation patterns in the PSD spectra in both fluorescent modified peptides at N-, C-terminal, and middle positions are considerably different, the common results of the remarkable enlargeness of the numbers of product ions by the fluorescent derivatization were realized. The increased numbers of the product ions by the fluorescently modification gave commonly great progress in the sequence analyses of peptides. This method will lead to a simple and easy application to complete sequence analysis of peptides with cysteine residue.

\section{Acknowledgment}

We thank Mr. Shin-ichirou Kawabata (Shimadzu, Japan) for his helpful discussion.

\section{References}

1) W. F. Patton, M. G. Pluskal, W. M. Skea, J. L. Buecker, M. F. Lopez, R. Zimmerman, L. M. Belanger, and P. D. Hatch, BioTechniques, 8, 518 (1990).

2) S. J. Cordwell, M. R. Wilkins, A. Cerpa-Poljak, A. A. Gooley, M. Duncan, K. L. Williams, and I. Humphery-Smith, Electrophoresis, 16, 438 (1995).

3) F. Kanda, S. Yoshida, T. Okumura, and T. Takamatsu, Rapid Commun. Mass Spectrom., 9, 1095 (1995).

4) P. R. Griffin, M. J. MacCoss, J. K. Eng, R. A. Blevins, J. S. Aaronson, and J. R. Yates, III, Rapid Commun. Mass 
Spectrom., 9, 1546 (1995).

5) B. Spengler, D. Kirsch, R. Kaufmann, and E. Jaeger, Rapid Commun. Mass Spectrom., 6, 105 (1992).

6) R. Kaufmann, B. Spengler, and F. Lutzenkirchen, Rapid Commun. Mass Spectrom., 7, 902 (1993).

7) M. Bartlet-Jones, W. A. Jeffery, H. F. Hansen, and D. J. C. Rappin, Rapid Commun. Mass. Spectrom., 8, 737 (1994).

8) B. Spengler, F. Luetzenkirchen, S. Metzger, P. Chaurand, R. Kaufmann, W. Jeffery, M. Bartlet-Jones, and D. J. C. Pappin, Int. J. Mass Spectrom. Ion Processes, 169/170, 127 (1997).

9) B. Spengler, J. Magn. Spectrom., 32, 1019 (1997).

10) S. Dikler, J. W. Kelly, and D. H. Russell, J. Mass Spec- trom., 32, 1337 (1997).

11) K. D. W. Roth, Z-H. Huang, N. Sadagopan, and J. T. Watson, Mass Spectrom. Rev., 17, 255 (1998).

12) M. Nakagawa, T. Yamagaki, and H. Nakanishi, Electrophoresis, 21, 1651 (2000).

13) K. Kuroda, M. Nakajima, H. Minakata, and Y. Hosaka, Virus Res., 38, 43 (1995).

14) M. Brinkley, Bioconjugate Chem., 3, 2 (1992).

15) K. Biemann, Biochem. Soc. Trans., 17, 237 (1989).

Keywords: MALDI-TOF-MS, PSD method, Peptide sequence, Fluorescently modification, Nucleoprotein in influenza virus 